\title{
Proteomics-based identification of a group of apoptosis-related proteins and biomarkers in gastric cancer
}

\author{
ZHIGANG BAI ${ }^{1,4^{*}}$, YINGJIANG YE ${ }^{1 *}$, BIN LIANG $^{1}$, FENG XU $^{1}$, HUI ZHANG ${ }^{1}$, YANBIN ZHANG $^{1}$, \\ JIAROU PENG ${ }^{2}$, DANHUA SHEN ${ }^{3}$, ZHIRONG CUI $^{1}$, ZHONGTAO ZHANG $^{4}$ and SHAN WANG ${ }^{1}$ \\ ${ }^{1}$ Department of Gastroenterological Surgery, Surgical Oncology Laboratory, Peking University People's Hospital, Beijing \\ 100044; ${ }^{2}$ Laboratory of Proteomics, Peking University, Beijing 100083; ${ }^{3}$ Department of Pathology, Peking University \\ People's Hospital, Beijing 100044; ${ }^{4}$ General Surgery Laboratory, Beijing Friendship Hospital, Beijing 100050, P.R. China
}

Received February 9, 2010; Accepted April 19, 2010

DOI: $10.3892 /$ ijo. 2010.873

\begin{abstract}
Gastric cancer (GC) is the one of the most common types of cancer in Asia. To better understand the molecular mechanisms underlying GC, and to seek new markers of tumor progression, we used a proteomics strategy to analyze the protein expression patterns in matched pairs of GC tissue and normal gastric mucosa of $8 \mathrm{GC}$ patients. Comparative proteomic analysis, using two-dimensional gel electrophoresis (2-DE) and matrix-assisted laser-desorption ionization timeof-flight mass spectrometry (MALDI-TOF-MS), revealed that 32 protein spots showed a $>2$-fold difference in intensity between tumor and normal tissues. Twenty-six proteins were up-regulated and 6 proteins were down-regulated in tumor tissue compared to control. Western blot analysis confirmed differential expression for 9 proteins, including AGR2, ENO1, GDI2, GRP78, GRP94, PPIA, PRDX1, PTEN and VDAC1. Immunohistochemical staining of a tissue microarray, derived from 145 GC patients, with antibodies for each of the 9 proteins demonstrated a significant association between the level of protein immunostaining and the clinical features of the disease in the donor. The identified proteins were functionally classified using bioinformatics methods, showing that the 9 proteins identified were related to BCL2,
\end{abstract}

Correspondence to: Dr Shan Wang, Department of Gastroenterological Surgery, Surgical Oncology Laboratory, Peking University People's Hospital, 11 Xizhimen South Road, Xi-Cheng District, Beijing 100044, P.R. China

E-mail: bjphbpc@yahoo.com.cn

Dr Zhongtao Zhang, Department of General Surgery, Beijing Friendship Hospital, Capital Medical University, 95 Yongan Road, Xuan-wu District, Beijing 100050, P.R. China

E-mail: zhangzht@yahoo.com.cn

*Contributed equally

Key words: gastric cancer, proteomics, tissue microarray, biomarkers, apoptosis
BAX, ERBB2 and CASP3 proteins and involved in the process of apoptosis. These proteomic data provide potentially valuable insights into both the biology of GC and the identity of biomarkers for tumor progression. We propose ENO1, GRP78, GRP94, PPIA, PRDX1 and PTEN as potential GC biomarkers.

\section{Introduction}

Gastric cancer (GC) is one of the most common malignancies, having the second highest mortality rate of all tumors and a 5-year survival rate of only $20 \%$ (1). GC patients are often at advanced stages when first diagnosed. A recent survey indicated that the 5-year survival rate of AJCC stage IV GC patients is only $7-10.1 \%$, while that for stage IA disease is between 78 and $93.3 \%$ (2,3). Early diagnosis of GC is thus critical for effective treatment. Unfortunately, for most patients, the diagnosis of GC is only made in the presence of significant clinical symptoms. Thus, the development of sensitive, specific and convenient methods for diagnosing GC could considerably improve our ability to treat this disease and reduce mortality (4).

At present, one or several combined tumor markers can be detected serologically, but the low sensitivity and specificity of these assays renders them unsuitable for use in epidemiological and clinical diagnostic screening (5). The first step in uncovering new and potentially useful biomarkers is to identify one or more candidates of interest, or even to identify a spectrum of potentially useful biomarkers. Such a strategy depends on a high-throughput screening method, for which proteomics technology is ideally suited (6). Proteomics technology can be used for comprehensive, dynamic, quantitative analysis and comparison of tumor cells and cells of normal origin, and in tissues at different stages of the disease. This can allow tumor-specific proteins involved in tumorigenesis, tumor differentiation and tumor metastasis to be identified. These proteins can not only provide clues to the pathogenesis of tumors, but can also be screened to identify biomarkers and tumor-specific antigens that can be used for early tumor diagnosis and treatment, as well is the identification of new therapeutic targets (7).

In the current study, we analyzed the protein expression profiles of poorly differentiated tumors and matched adjacent 
normal tissue from 8 surgical GC patients. Our objective was to seek proteins related to gastric cancer, to analyze and functionally classify proteins using systems biology methods, to use immunohistochemical analysis of tissue arrays to screen gastric cancer-related proteins, and to study the relationship between those cancer-related protein proteins and clinical pathological characteristics of GC.

\section{Materials and methods}

Gastric tissue samples. Eight pairs of GC tissue and adjacent non-cancerous gastric mucosa were obtained from Beijing People's Hospital (Beijing, China). Care was taken to obtain cancer tissue samples from the core area of the tumor, so as to avoid inclusion of adjacent non-cancerous tissue. For adjacent normal tissue, epithelium at least $5 \mathrm{~cm}$ from the border of the GC was selectively excised, with care again taken to minimize contamination by non-epithelial cells. The samples were washed with physiological saline to remove contaminants and then frozen in liquid nitrogen. Diagnosis of GC was confirmed histopathologically after HE staining, and the samples were classified according to Lauren's classification (8). All cancer tissue specimens were histologically diagnosed as advanced cancer. Patient information is listed in Table I. This study was approved by the Ethics Committee of Peking University People's Hospital.

Sample preparation. Tissue $(50 \mathrm{mg})$ was crushed using a metal mortar immersed in liquid nitrogen, and then precipitated with $10 \%$ TCA/acetone for $2 \mathrm{~h}$. The precipitate was washed twice with precooled acetone. After removing the acetone by vacuum evaporation, the pellet was dissolved in lysis buffer containing $7 \mathrm{M}$ urea, $2 \mathrm{M}$ thiourea, 4\% 3-[(3-cholamidopropyl) dimethylammonio]-1-propanesulfonate (CHAPS), 0.2\% Bio-Lyte (pH 3.0-10 L), 65 mM dithiothreitol (DTT). The lysate was sonicated with a probe sonicator for $5 \mathrm{~min}$, followed by centrifugation at $40,000 \mathrm{x} g$ for $30 \mathrm{~min}$. After the quantitative measurement of protein concentration by Bradford assay, the supernatant was stored at $-80^{\circ} \mathrm{C}$ until use.

Protein separation by two-dimensional gel electrophoresis $(2-D E)$. Protein (approximately $100 \mu \mathrm{g}$ ) was loaded onto an IPG strip (pH 3.0-10 L, $17 \mathrm{~cm}$, Bio-Rad) and subjected to isoelectric focusing in the Protean IEF cell system (Bio-Rad). Briefly, the strips were rehydrated for $4 \mathrm{~h}$ at $0 \mathrm{~V}$ and for $8 \mathrm{~h}$ at $50 \mathrm{~V}$. Isoelectric focusing was carried out using a program of graded voltage consisting of 500, 1000 and $8000 \mathrm{~V}$, each for $1 \mathrm{~h}$, with voltage then remaining at $8000 \mathrm{~V}$ until the $\mathrm{Vh}$ reached 60,000 . The focused strips were reduced with $1 \%$ DTT and alkylated with $2.5 \%$ iodoacetamide (IAM) in buffer containing $6 \mathrm{M}$ urea, $50 \mathrm{mM}$ Tris- $\mathrm{HCl}$ (pH 8.8), 30\% glycerol, $2 \%$ SDS, and trace bromophenol blue. For secondary electrophoresis, the treated strips were subjected to $12 \%$ SDSPAGE $(200 \mathrm{~mm})$ in a Protean II xi Cell (Bio-Rad). The proteins on the 2-DE gels were visualized using silver staining and Coomassie Brilliant Blue R250 staining (1 mg protein was loaded). All samples were analyzed in duplicate.

Comparative image analysis of 2-DE spots. All 2-DE gels were scanned at 300 dpi using a DuoScan T1200 scanner
(AGFA). Relative spot volumes were estimated using ImageMaster 2-D Platinum software, version 5.0 (GE Healthcare). To minimize differences in protein concentration among the samples from different patients, mixed pools, containing equal amounts of protein from each sample, either the GC or the adjacent tissue, were prepared, run on 2-DE gels and set as the reference. In addition, all spots with differing intensity between normal and GC tissue were rechecked in 8 pairs of 2-DE images from each sample pair, to identify those with a high incidence rate. A significant difference in the expression of a protein between tumor and normal tissue was defined as $>2$-fold change in spot density in $>30 \%$ of tumor specimens (9).

Protein identification by mass spectrometry. The differentially expressed spots were manually excised from gels and transferred into microfuge tubes. The gel particles were subjected to in-gel digestion with $0.01 \mu \mathrm{g}$ of trypsin (Sigma) at $37^{\circ} \mathrm{C}$ overnight. The peptides generated from tryptic digestion were spotted onto Anchorchip (Bruker), and cocrystallized with cyano-4-hydroxycinnamic acid (CHCA) $(4 \mathrm{mg} / \mathrm{ml})$. The mass spectra of peptides were obtained using an Ultraflex matrix-assisted laser-desorption ionization timeof-flight mass spectrometry (MALDI-TOF-MS) (Bruker). Peptide mass fingerprints were performed using MASCOT search (http://www.matrixscience.com). Probability scores above 58 in the MASCOT search were defined as significant.

Ingenuity pathway analysis (IPA). The ingenuity pathways knowledge base (Ingenuity System, Mountain View, CA) is a large, curated database consisting of millions of individually modeled inter-protein relationships culled from the biological literature. Proteins that appeared to be differentially expressed between GC tissue and adjacent gastric mucosa were analyzed using IPA5.0 to determine the most relevant biological mechanisms, interaction networks and functions that applied to them. Proteins that were under-expressed in GC as well as those that were over-expressed were subjected to this analysis.

Western blot analysis. To isolate total protein, frozen tissue samples were lysed in RIPA lysis buffer $(50 \mathrm{mM}$ Tris- $\mathrm{HCl}$ (pH 7.5), $50 \mathrm{mM} \mathrm{NaCl}, 1 \%$ Nonidet $\mathrm{P} 40,1 \%$ sodium deoxycholate, $0.1 \%$ SDS and 2 mM EDTA) with $1 \%$ protease inhibitor cocktail (Amerco). Protein $(50 \mu \mathrm{g})$ was separated by $12 \%$ SDS-PAGE and then transferred onto polyvinylidene fluoride membranes (Millipore). After treating with 5\% nonfat dry milk in TBST (25 mM Tris- $\mathrm{HCl}, \mathrm{pH} 7.5,150 \mathrm{mM}$ $\mathrm{NaCl}$ and $0.05 \%$ Tween-20) for $2 \mathrm{~h}$ at room temperature, the membranes were incubated overnight at $4^{\circ} \mathrm{C}$ with primary antibody (AGR2, PRDX1, Abcam; ENO1, GDI2, GRP94, PPIA, Protein Tech Group Inc; GRP78, Santa Cruz; or PTEN, Zymed), followed by horseradish-peroxidase-conjugated secondary antibody (Santa Cruz) for $1 \mathrm{~h}$ at room temperature. GAPDH (Santa Cruz) was used as an internal control. Target proteins were detected using an ECL kit (GE Healthcare) and exposure to X-ray film (Kodak).

Tissue samples and tissue microarray (TMA). A group of 145 consecutive patients with GC were studied. All patients had been treated by radical D2 gastrectomy in the Peking 
Table I. Clinical features of the patients with gastric cancer.

\begin{tabular}{|c|c|c|c|c|c|c|}
\hline Case & Gender & Age & Location & Diameter $(\mathrm{cm})$ & Differentiated & pTNM \\
\hline 1 & Male & 75 & Corpus & 13 & Poor & T4N2M1 \\
\hline 2 & Female & 38 & Antrum & 4 & Poor & T3N1M0 \\
\hline 3 & Male & 65 & Atrum & 6 & Poor & T3N1M0 \\
\hline 4 & Male & 78 & Antrum & 4 & Moderate & T3N1M0 \\
\hline 5 & Male & 68 & Antrum & 5 & Poor & T3N1M0 \\
\hline 6 & Male & 67 & Antrum & 3 & Poor & T3N2M0 \\
\hline 7 & Female & 34 & Antrum & 6 & Poor & T4N2M0 \\
\hline 8 & Male & 51 & Antrum & 4 & Poor & T4N1M0 \\
\hline
\end{tabular}

$\mathbf{A}$

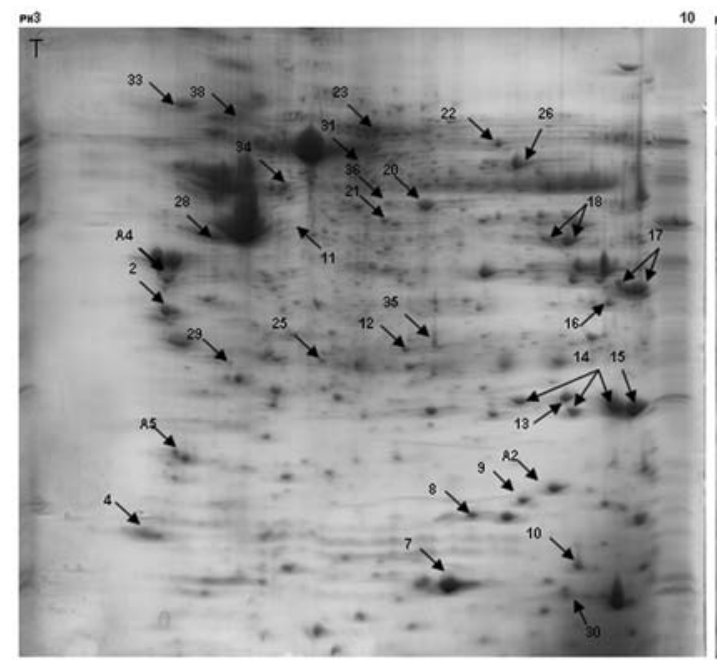

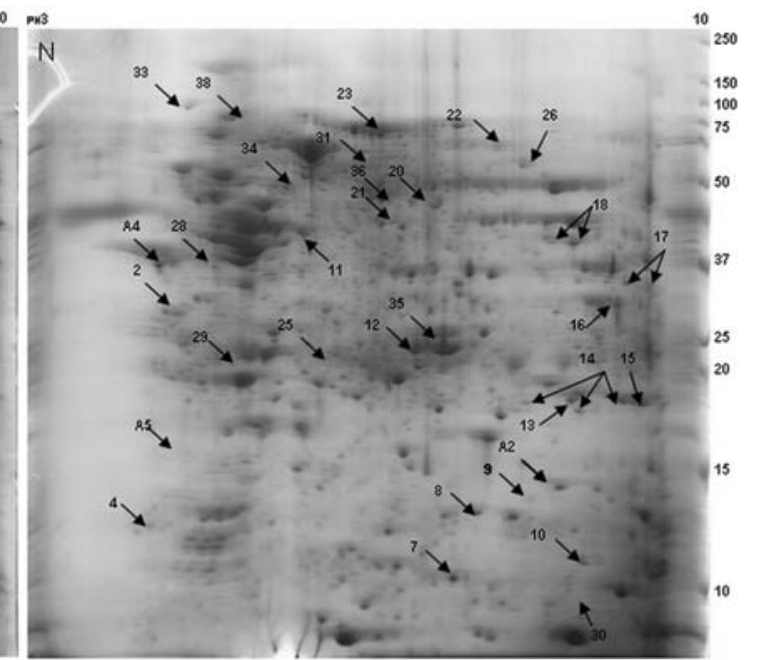

Figure 1. 2-D protein profiles from human gastric cancer and adjacent normal tissues. Proteins were separated on the basis of pI (X-axis) and molecular mass (Y-axis) and visualized by silver staining. Each marked protein was identified by MALDI-TOF MS. (A) 2-DE pattern from gastric cancer tissues. (B) 2-DE pattern from normal tissues. Labeled spots indicate proteins down-regulated in carcinoma tissue. Arrows indicate each protein spot. These proteins exhibited a significant difference in expression level from that in normal tissue in $>40 \%$ of tumor patients.

University People's Hospital between January 1999 and June 2002. Clinical and pathological information for this group of patients was obtained by review of histopathology reports and medical records. GC stage was assessed according to the 2002 tumor-node-metastasis (TNM) classification of malignant tumors by the American Joint Committee on Cancer (AJCC). The patients in the study were followed by direct evaluation or phone interview until their death or June 2007, which provided a minimum of 5 years of follow-up. Tissue microarrays were constructed as previously described (10).

Immunohistochemistry. Immunohistochemistry was carried out as previously described (10). Briefly, the slides were deparaffinized, rehydrated and treated with $3 \%$ hydrogen peroxide. After antigen retrieval, the sections were incubated with primary antibodies overnight at $4^{\circ} \mathrm{C}$. Primary antibodies were detected using the Powervision two-step histostaining reagent (Zhongshan, Beijing), with PV-6001 as the secondary antibody, and detection was by the diaminobenzidine (DAB) chromogenic reaction. Tissues were counterstained, dehydrated and mounted. Positive and negative controls were included. Two experienced pathologists who were blind to the patients' clinical history independently examined the stained slides.
Statistical analysis. All data were analyzed using SPSS11.0 software. The association of protein expression with various clinicopathological features was analyzed using the $\chi^{2}$ test and the leave-one-out cross validation (LOOCV) test. Cumulative survival was estimated by the Kaplan-Meier method, and differences between survival curves were analyzed using a logrank test. $\mathrm{P}<0.05$ was considered to be statistically significant.

\section{Results}

Proteins differentially expressed between GC tissues and adjacent gastric mucosa. To identify proteins that might be differentially expressed in GC, we processed 8 pairs of GC tissue and adjacent normal tissues using 2-DE. Approximately 900 protein spots were detected by silver staining. We obtained reproducible 2-DE profiles and relative spot intensities from all samples when we performed the experiments in duplicate. Only spots that had a $>2$-fold difference in density between normal and GC tissue were classified as down- or up-regulated. We identified 26 protein spots that were up-regulated in GC, and 6 protein spots that were downregulated (Fig. 1). These proteins were selected for mass spectrum (MS) analysis, and identified using peptide mass 
Table II. Proteins with altered expression in gastric cancer and their identification by MS.

\begin{tabular}{|c|c|c|c|c|c|c|}
\hline & \multirow[b]{2}{*}{ Protein } & \multirow[b]{2}{*}{ SwissProt } & \multirow[b]{2}{*}{$\begin{array}{l}\text { Mascot } \\
\text { score }\end{array}$} & \multirow[b]{2}{*}{$\begin{array}{l}\text { Nominal mass } \\
\text { (Mr), calculated } \\
\text { pI value }\end{array}$} & \multicolumn{2}{|c|}{ No. of mass values matched } \\
\hline & & & & & Sequence & $\begin{array}{c}\text { Coverage } \\
(\%)\end{array}$ \\
\hline \multicolumn{7}{|c|}{ Up-regulation } \\
\hline 4 & MYL6_HUMAN & P60661 & 89 & $16788,4.46$ & 9 & 60 \\
\hline 7 & HBB_HUMAN & P68871 & 135 & $15853,6.81$ & 12 & 84 \\
\hline 8 & PPIA_HUMAN & P62937 & 62 & $17870,7.82$ & 5 & 32 \\
\hline 9 & DEST_HUMAN & P60981 & 83 & $18362,8.11$ & 1 & 8 \\
\hline 10 & PROF1_HUMAN & P07737 & 70 & $14914,8.48$ & 5 & 41 \\
\hline 12 & PGAM1_HUMAN & P18669 & 155 & $28655,6.75$ & 15 & 50 \\
\hline 13 & PRDX1_HUMAN & Q06830 & 133 & $22096,8.27$ & 13 & 48 \\
\hline 14 & TAGLN2_HUMAN & P37802 & 130 & $22246,8.45$ & 13 & 61 \\
\hline 15 & TAGL_HUMAN & Q01995 & 205 & $22465,8.88$ & 18 & 71 \\
\hline 16 & VDAC1_HUMAN & P21796 & 169 & $30623,8.63$ & 17 & 60 \\
\hline 17 & CNN1_HUMAN & P51911 & 152 & $33150,9.14$ & 17 & 49 \\
\hline 18 & ALDOA_HUMAN & P04075 & 117 & $39264,8.39$ & 14 & 40 \\
\hline 20 & EFTU_HUMAN & P49411 & 142 & $49510,7.26$ & 17 & 42 \\
\hline 21 & ENOA_HUMAN & P06733 & 161 & $47008,6.99$ & 17 & 47 \\
\hline 25 & HSPB1_HUMAN & P04792 & 98 & $22768,5.98$ & 11 & 55 \\
\hline 28 & ACTH_HUMAN & P63267 & 109 & $41850,5.31$ & 13 & 37 \\
\hline 29 & GDIS_HUMAN & P52566 & 128 & $22974,5.10$ & 13 & 73 \\
\hline 30 & FKB1A_HUMAN & P62942 & 70 & $11812,8.07$ & 7 & 44 \\
\hline 31 & AL1A1_HUMAN & P00352 & 87 & $54696,6.29$ & 13 & 33 \\
\hline 33 & ENPL_HUMAN & P14625 & 170 & $92411,4.76$ & 16 & 19 \\
\hline 35 & CAH2_HUMAN & P00918 & 166 & $29097,6.86$ & 15 & 55 \\
\hline 36 & AGR2_HUMAN & O95994 & 62 & $19967,9.03$ & 5 & 33 \\
\hline 38 & GRP78_HUMAN & P11021 & 161 & $72288,5.07$ & 14 & 28 \\
\hline A2 & COF1_HUMAN & P23528 & 68 & $18360,8.26$ & 6 & 33 \\
\hline A4 & TPM2_HUMAN & P07951 & 374 & $32970,4.63$ & 8 & 24 \\
\hline A5 & MYL9_HUMAN & Q6IBG1 & 159 & $19814,4.80$ & 4 & 29 \\
\hline \multicolumn{7}{|c|}{ Down-regulation } \\
\hline 2 & TPM4_HUMAN & P67936 & 79 & $28373,4.67$ & 11 & 42 \\
\hline 11 & PTEN_HUMAN & P60484 & 89 & $47700,5.85$ & 18 & 33 \\
\hline 22 & TKT_HUMAN & P29401 & 166 & $67835,7.58$ & 20 & 34 \\
\hline 23 & TRFE_HUMAN & P02787 & 125 & $76996,6.81$ & 19 & 27 \\
\hline 26 & KPYM_HUMAN & P14618 & 215 & $57769,7.95$ & 23 & 48 \\
\hline 34 & K2C8_HUMAN & P05787 & 245 & $53510,5.52$ & 29 & 52 \\
\hline
\end{tabular}

fingerprint (PMF) of the selected spots followed by a database search (Table II).

Classification and functional annotation of differentially expressed protein. We used ingenuity pathways analysis (IPA) software for functional classification and pathway analysis of the differentially expressed proteins. The 32 such proteins were divided into three groups according to their potential functions in GC, which included both cell death and tumor cell morphology (Table III). IPA pathway analysis indicated that nine proteins, related to BCL2, BAX, ERBB2 and
CASP3, had roles relating to apoptosis (Fig. 2). This group comprised AGR2 (anterior gradient homolog 2), ENO1 (enolase 1), GDI2 (Rho GDP dissociation inhibitor ß), GRP78 (glucose-regulated protein 78), GRP94 (glucose-regulated protein 94), PPIA (peptidylprolyl isomerase A), PRDX1 (peroxiredoxin 1), PTEN (phosphatase and tensin homolog), and VDAC1 (voltage-dependent anion channel 1). Although ENO1 (11), GDI2 (12), GRP78 (13), GRP94 (14) and PTEN (15) have each been previously implicated in GC, no such connection has previously been established for AGR2, PPIA, PRDX1, or VDAC1. 
A

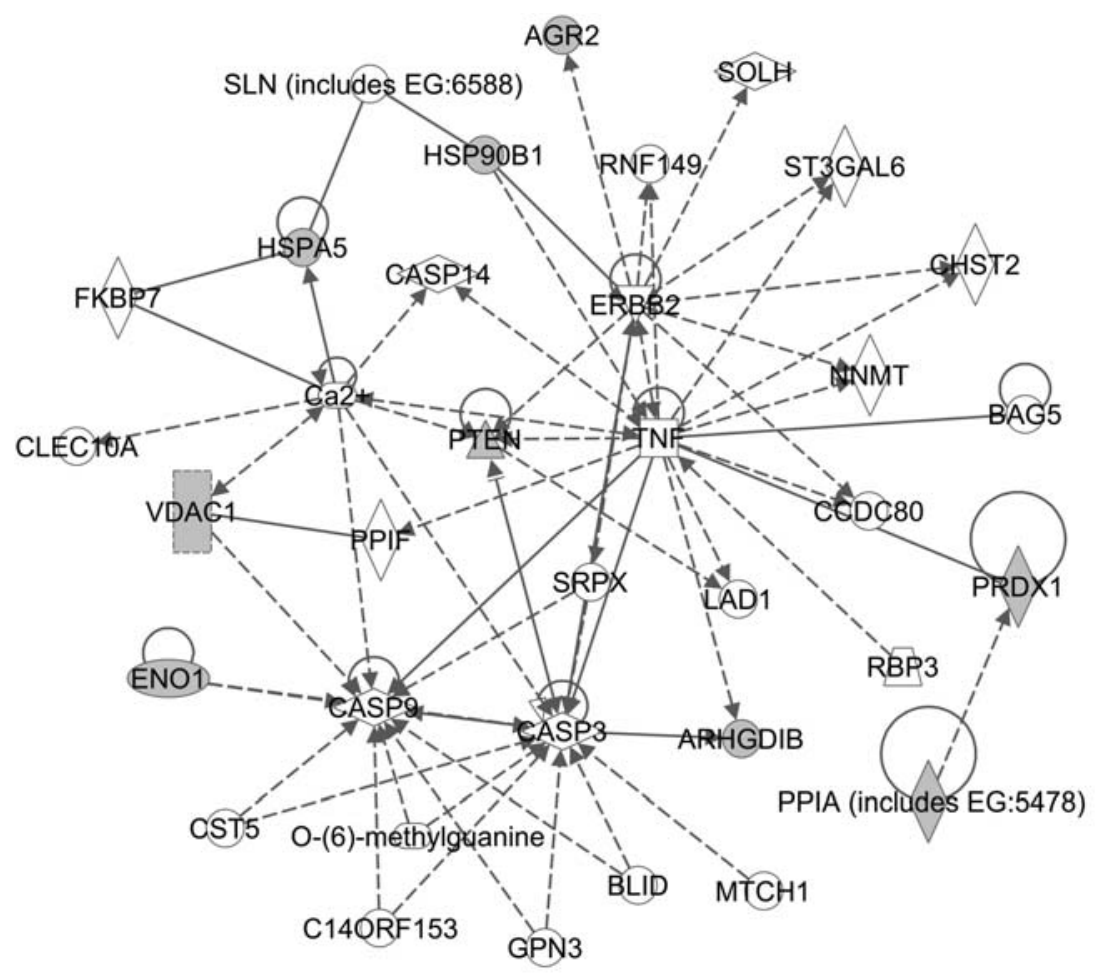

B

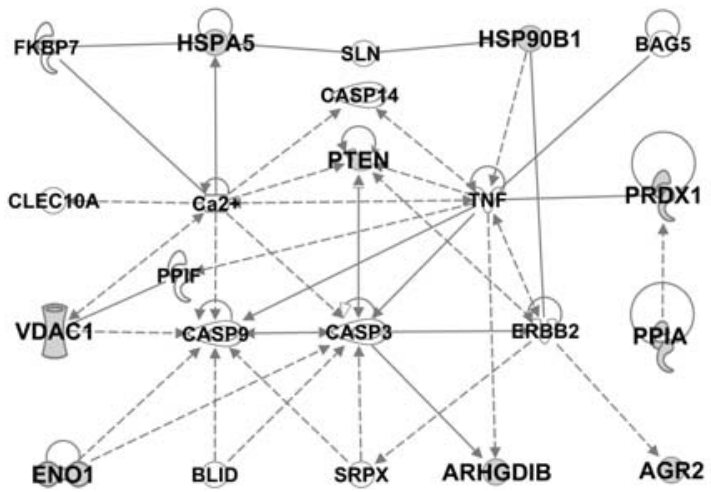

$\checkmark$ Cytokine/Growth Factor

- Chemical/Toxicant

B Enzyme

7. Ion Channel

\&o Kinase

(a) Peptidase

๖ Phosphatase

๑ Transcription Regulator

$\nabla$ Transporter

Unknown

- Relationship

-- Relationship

Figure 2. A network of functionally related proteins based on differential expression in GC compared to adjacent normal gastric mucosa. The network represents proteins assigned by Ingenuity software as being involved in cell death. Light gray shading indicates up-regulation; dark grey shading indicates down-regulation. The shape of each node indicates function: diamonds designate an enzyme; square, growth factor; triangle, kinase; circle, other. The style of each connecting line indicates the nature of the interaction between the nodes it joins: - indicates a physical interaction; $\rightarrow$ indicates functional interaction (activation); - indicates inhibition. A, global map; B, sketch.

Table III. Function grouping of proteins with altered expression in gastric cancer (ingenuity pathway analysis).

\begin{tabular}{|c|c|c|c|c|}
\hline Team & Molecules in network & Top functions & Score & $\begin{array}{c}\text { Focus } \\
\text { molecules }\end{array}$ \\
\hline 1 & $\begin{array}{l}\text { AGR2, CNN1, HBB, ENPL(GRP94), } \\
\text { GRP78, KRT8(K2C8), MYL6, MYL9, } \\
\text { PGAM1, PTEN, TAGL, TPM2, TPM4, } \\
\text { TUFM(EFTU) }\end{array}$ & $\begin{array}{l}\text { Cell death, cellular reproductive } \\
\text { movement, reproductive system } \\
\text { development and function }\end{array}$ & 32 & 14 \\
\hline 2 & $\begin{array}{l}\text { ACTH, AL1A1, ALDOA, CAH2, COF1, } \\
\text { DEST, ENO1(ENOA), PROF1, KPYM, } \\
\text { PPIA, PRDX1, TRFE, TKT, VDAC1 }\end{array}$ & $\begin{array}{l}\text { Cancer, cell death, reproductive } \\
\text { system disease }\end{array}$ & 32 & 14 \\
\hline 3 & GDIS(GDI2), FKB1A, HSPB1, TAGLN2 & $\begin{array}{l}\text { Cell death, neurological disease, } \\
\text { cell morphology }\end{array}$ & 7 & 4 \\
\hline
\end{tabular}


A

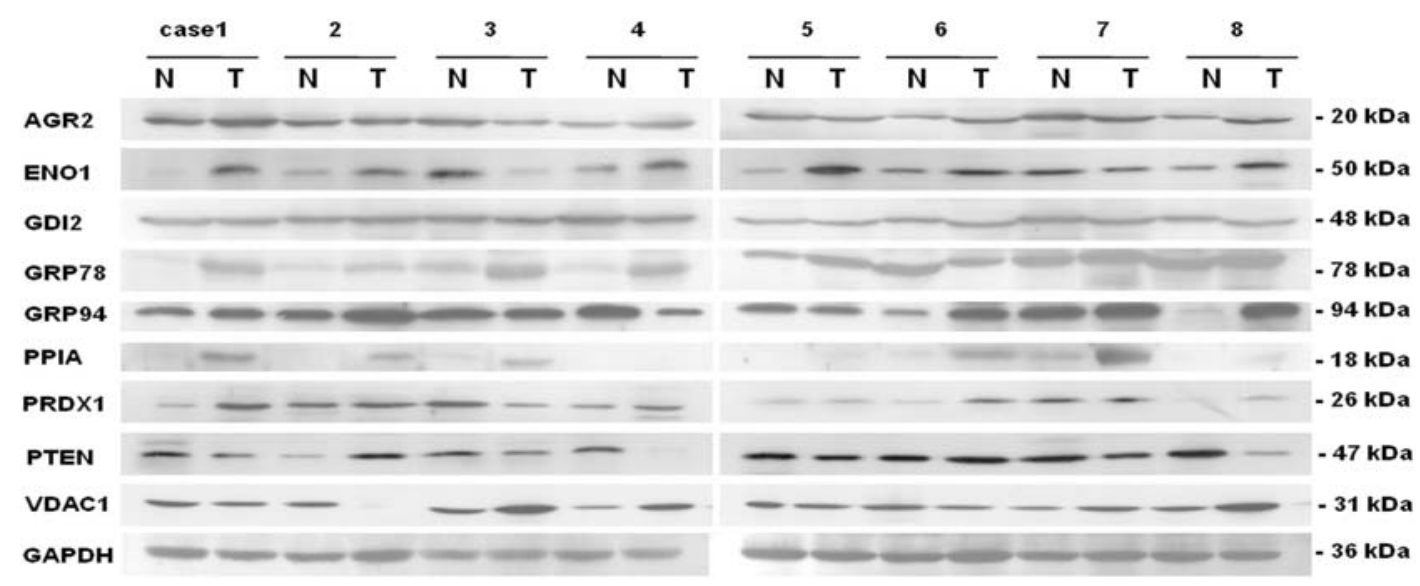

B

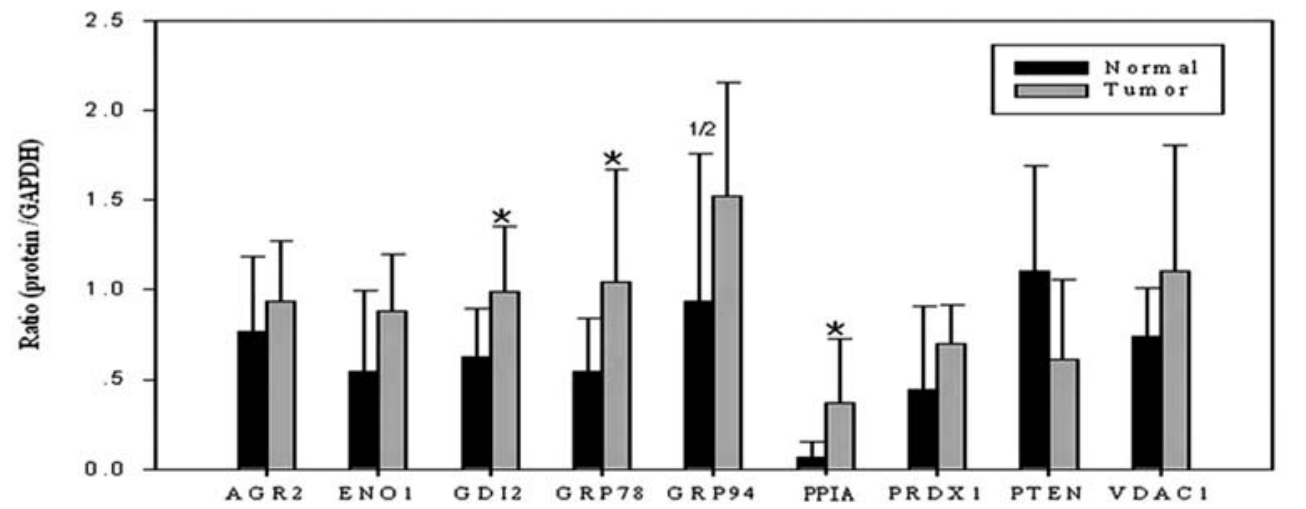

Figure 3. (A) Proteins differentially expressed in gastric cancer tissues and matched adjacent mucosa. Western blot analysis for validating decreased expression of PTEN in gastric cancer tissues and the increased expression of AGR2, ENO1, GDI2, GRP78, GRP94, PPIA, PRDX1 and VDAC1. GAPDH was used as reference. (B) Grayscale scanning of Western blot bands. The ratio of grayscale value of each differentially altered protein compared to GAPDH was statistically analyzed. All proteins other than PTEN are highly expressed in tumor tissue. The expression of GDI2, GRP78 and PPIA are significantly different between gastric cancer and normal tissue.

Expression of nine apoptosis-related proteins in GC. To examine the expression level of these proteins, Western blot analysis was carried out using the tissue specimens from which the 2-DE samples were derived. As expected, PTEN was found to be consistently suppressed in tumor tissue, whereas each of the other eight proteins were highly expressed (Fig. 3A). There were significant differences between $\mathrm{GC}$ tissue and adjacent normal tissue in the expression of GDI2, GRP78 and PPIA (Fig. 3B). These results were consistent with the results of two-dimensional gel electrophoresis.

Expression of differential proteins in GC TMA. To further investigate whether AGR2, ENO1, GDI2, GRP78, GRP94, PPIA, PRDX1 and PTEN are expressed in GC tissues, and to determine which cells express these proteins, immunohistochemical analysis was performed using tissue microarrays.

Expression of AGR2, ENO1, GDI2, GRP78, GRP94, PPIA and PRDX1 was evident in the cytoplasm of both GC cells and non-tumor cells. However, in each case there was more intense staining of the GC cells than non-tumor cells. It was also apparent that AGR2 was expressed in the extracellular space, and that GDI2 was not significantly expressed in non-tumor cells (Fig. 4A).

In contrast, PTEN showed almost no expression in the cytoplasm of GC cells and epithelial cells, but was instead expressed in the nuclei of some columnar epithelial cells.
This expression pattern was termed 'nuclear PTEN'. The expression of all other proteins was increased in tumor tissue (Fig. 4A). Collectively, significant differences in expression level between tumor and normal tissues were seen for AGR2, ENO1, GRP78, GRP94, PRDX1 and nuclear PTEN ( $\mathrm{P}=0.001$, $<0.001,0.002,<0.001,0.034$ and 0.027 , respectively).

Differential protein expression is correlated with the clinical features of GC patients. We analyzed the potential relationship between the expression of the 8 apoptosis-related proteins and the prognosis of GC patients. The results indicated that the prognosis of GC patients with positive expression of nuclear PTEN was more favorable than for those patients with negative PTEN expression $(\mathrm{P}=0.016)$. The prognosis of GC patients with positive expression of ENO1, GRP78, GRP94, PPIA and PRDX1 tended to be worse than that of patients with negative expression of these proteins $(\mathrm{P}=0.002$, $\mathrm{P}=0.006, \mathrm{P}<0.001, \mathrm{P}=0.047, \mathrm{P}=0.034$, respectively, Fig. 4B). LOOCV evaluations revealed that the GC prediction rate using the 8 apoptosis-related proteins qualified each of them as GC biomarkers, with a combined-biomarker accuracy of $70 \%$.

\section{Discussion}

The biomarkers most commonly used for the diagnosis and postoperative follow-up of GC patients are carcinoembryonic 
A

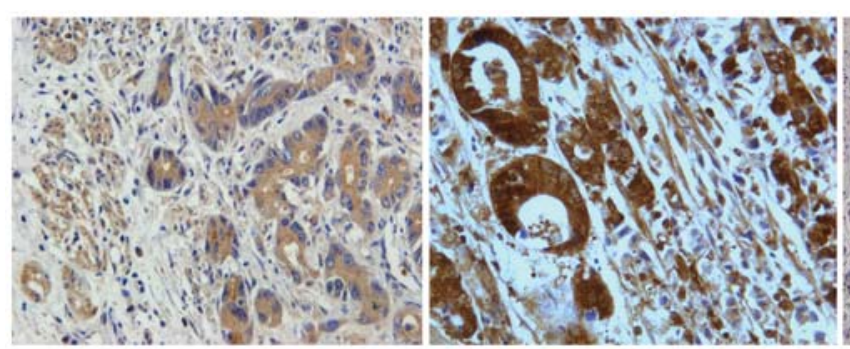

AGR2

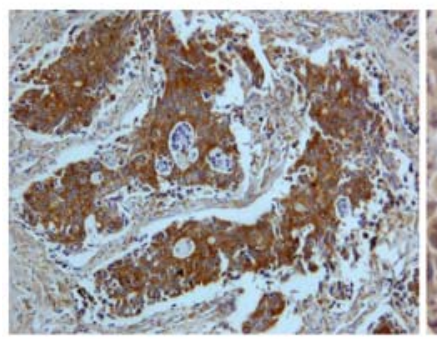

GRP94

B

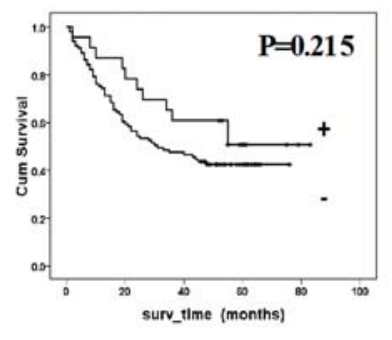

AGR2

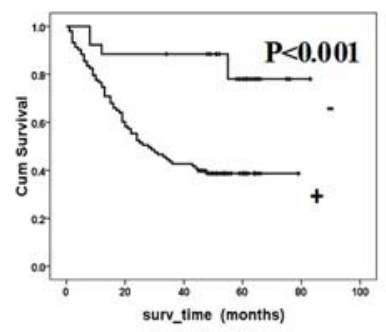

GRP94
ENO1

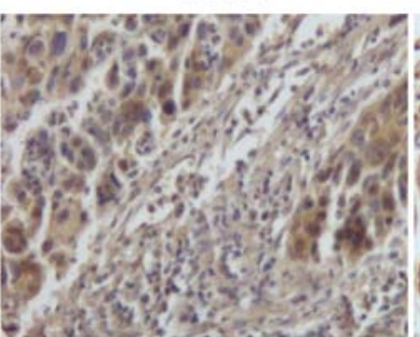

PPIA

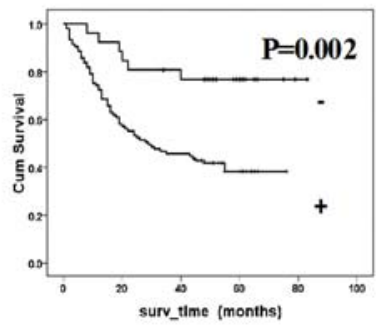

ENO1

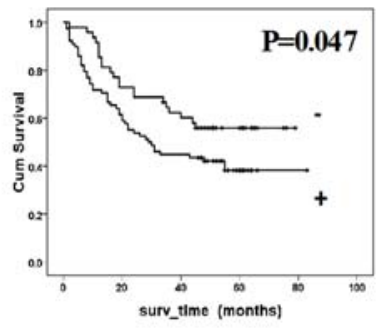

PPIA

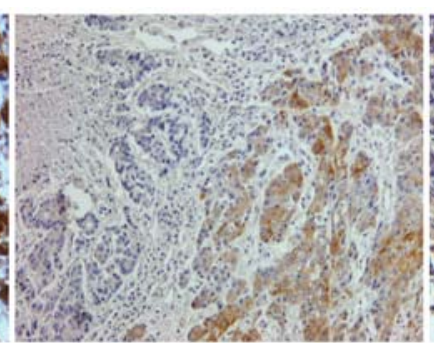

GDI2

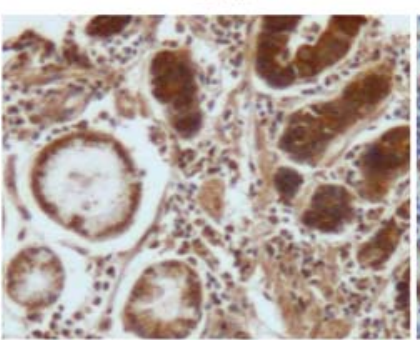

PRDX1

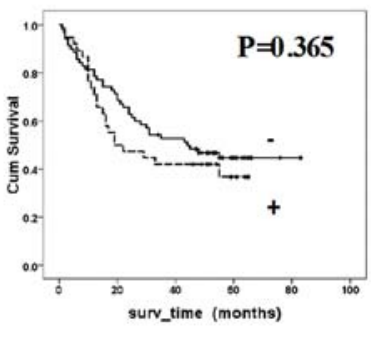

GDI2

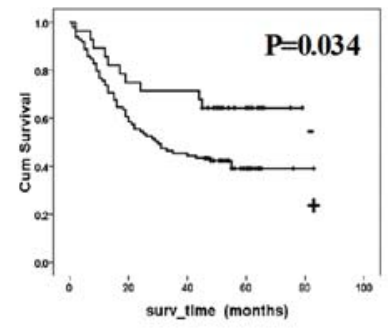

PRDX1

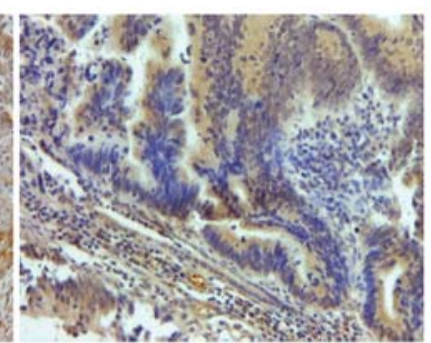

GRP78

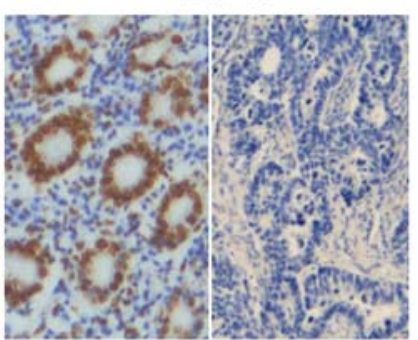

PTEN

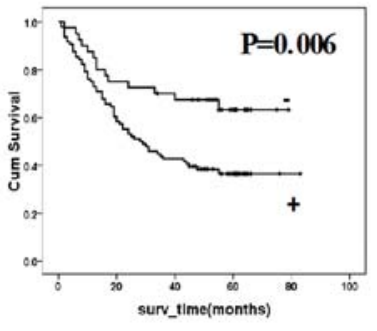

GRP78

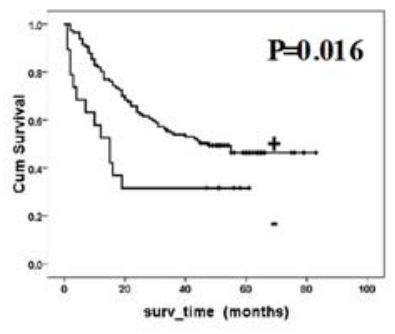

PTEN

Figure 4. (A) Immunohistochemical staining of gastric cancer and adjacent normal tissue sections. Sections were immunostained with antibodies against AGR2, ENO1, GDI2, GRP78, GRP94, PPIA, PRDX1 and PTEN proteins. All proteins other than PTEN are highly expressed in tumor tissue. Brown color indicates positive immunoreactivity. (B) Kaplan-Meier survival curves, for 145 gastric cancer patients receiving radical D2 gastrectomy, categorized by AGR2, ENO1, GDI2, GRP78, GRP94, PPIA, PRDX1 and PTEN expression.

antigen (CEA), carbohydrate antigen (CA19.9) and CA72-4. Although several new biomarkers for this disease have recently been identified, none has proven to be sufficiently sensitive and specific to be of reliable use for GC diagnosis (16). In the present study, we used a proteomic approach to identify proteins that are differentially expressed in human GC tissues versus the adjacent gastric mucosa. We identified 26 proteins that were up-regulated and 6 proteins that were down-regulated in GC tissue, providing a potentially rich source of research material from which to identify additional biomarkers.

Recently, rapid development of proteomics research strategies, integrated with sophisticated bioinformatics tools, allows thousands of candidate proteins to be efficiently and simultaneously collected, processed, compared, analyzed, stored and disseminated. For each of the 32 differentially expressed proteins we identified, we used IPA software to the functional classification and the pathway to which they belong. Of the 32 proteins we isolated, 9 proved to be related to apoptosis. To verify these results, we used Western blotting with antibodies to each of the proteins we isolated to determine their comparative expression levels in GC and normal tissue, the results of which were consistent with the 2-DE data.

Zhang et al (17) reviewed GC proteomics research $(9,18$ 20) and found that different studies using this approach rarely 
reported the same set of proteins to be differentially expressed, a finding that may be related to differences in the source of the specimens, or the techniques of sample preparation and 2-DE. This suggests that it is particularly important to increase the sensitivity and repeatability of 2-DE and to confirm differential expression profiles in more subjects.

The tissue microarray technology is a high-throughput tool that enables a rapid and concurrent analysis of molecular targets in large numbers of specimens at the DNA, RNA and protein levels under standardized conditions, and has provided a valuable complement to proteomics. Using this technology, combined with immunohistochemical analysis, we analyzed 145 pairs of tissue samples from GC patients. The results not only confirmed the previous 2-DE and immunoblotting data, but allowed us to compare the relationship between the tissue expression of each of the proteins we identified and the clinical features of the disease from which each tissue sample was derived.

Each of the proteins we identified appears to have individual but overlapping roles. Secretion of AGR2 has been shown to be involved in cancer formation and cell movement, and to promote tumor growth, cell migration and cellular transformation (21), and in breast cancer has been correlated with metastasis and poor prognosis $(22,23)$. AGR2, AGR2 is also expressed at high levels in prostate cancer, where it has been considered for use as a biomarker (24). ENO1 has been shown to bind with the $c-m y c$ promoter, acting as a transcription repressor, and may be tumor suppressor gene (25). ENO1 has been shown to be upregulated in lung cancer (26), hepatocellular carcinoma (27) and GC (11).

GDI2 controls the access of Rho GTPases to regulatory guanine nucleotide exchange factors and GTPase-activating proteins (28), and may also play a role in tumor cell apoptosis (29). A recent survey showed that RhoGDI2 is involved in gastric tumor growth and metastasis, and that RhoGDI2 may be a useful marker for tumor progression of human gastric cancer (12). It should be noted that the GDI2 expression levels we observed in the tissue microarray were not significantly between normal and disease tissues so this finding should be further verified.

GRP78 promotes cellular protein maturation, and appears to be involved in tumor cell proliferation and migration (30). The expression of high levels of GRP78 in GC tumors is associated with poorer prognosis, secondary to chemotherapeutic drug resistance and anti-apoptotic effects (13). GRP94 is also involved in the maturation of synthesized proteins, participating protein folding, anti-apoptosis and protein transport, as well as in the cellular response to oxygen depletion (31).

Changes in PPIA phosphorylation activity have been shown to play important roles in the occurrence and development of human tumors. Although high levels of expression of PPIA have been documented in hepatocellular cancer (32), ours is the first report implicating it in GC. PRDX1 has been shown to act by scavenging oxygen-free radicals and is important for protecting against tumorigenesis. Dysfunction of the antioxidant enzyme defense system and active oxygen scavenging have been shown to cause oxidative damage to DNA and abnormal of cell division, and to result in development and progression (33). VDAC1 has been implicated in the formation of the permeability transition pore complex (PTPC), promoting the release of the mitochondria product which triggered apoptosis, as well as interacting with BCL2 during apoptosis $(34,35)$.

PTEN has been shown to be an important tumor suppressor gene, with abnormal expression correlated with apoptosis, proliferation and metastasis of tumor cells. In GC patients, cytoplasmic expression of PTEN is associated with better prognosis (15). Recent evidence suggests that the expression of nuclear PTEN may be related to the stability of chromosomes (36). In this study, there were distinct differences in nuclear PTEN expression between GC and matched normal tissue. We found nuclear expression of PTEN to be correlated with lymph node metastasis in GC, and that GC patients with high expression of nuclear PTEN had better prognosis.

The analysis of protein expression levels alone is insufficient to reliably identify valid protein markers. For example, Li et al reported that SM22, a commonly used tumor marker in the past was actually a sign of neovascularization, and had been identified on the basis of contamination of tumor samples with vascular endothelial cells (37). To avoid this scenario, we took care in the present study, to conform 2-DE results using immunohistochemistry and tissue microarray analysis.

Tissue microarray technology is a powerful tool for efficiently scanning a range of potential tumor biomarkers (38). Combined 2-DE, mass spectrometry, systems biology software and tissue microarray technology, we have identified and initially characterized ENO1, GRP78, GRP94, PPIA, PRDX1 and PTEN as potential tumor markers. The relationship between the expression of these proteins and the clinicopathological characteristics of GC patients merits further study.

\section{Acknowledgements}

This study was supported by a Doctoral Fund from the Ministry of Education of China (no. 200800100098).

\section{References}

1. Murray C and Lopez A: Alternative projections of mortality and disability by cause 1990-2020: Global burden of disease study. Lancet 349: 1498-1504, 1997.

2. Hundahl S, Phillips J and Menck H: The national cancer data base report on poor survival of U.S. gastric carcinoma patients treated with gastrectomy: American joint committee on cancer staging, proximal disease, and the different disease hypothesis. Cancer 88: 921-932, 2000.

3. Ushijima T and Sasako M: Focus on gastric cancer. Cancer Cell 5: 121-125, 2004.

4. Srinivas P, Kramer B and Srivastava S: Trends in biomarker research for cancer detection. Lancet Oncol 2: 698-704, 2001.

5. Ludwig $\mathrm{J}$ and Weinstein $\mathrm{J}$ : Biomarkers in cancer staging, prognosis and treatment selection. Nat Rev Cancer 5: 845-856, 2005.

6. Tyers M and Mann M: From genomics to proteomics. Nature 422: 193-197, 2003.

7. Ransohoff D: Cancer: developing molecular biomarkers for cancer. Science 299: 1679-1680, 2003.

8. Lauren P: The two histological main types of gastric carcinoma: Diffuse and so-called intestinal-type carcinoma. An attempt at a histo-clinical classification. Acta Pathol Microbiol Scand 64: 31-49, 1965.

9. Nishigaki R, Osaki M, Hiratsuka M, et al: Proteomic identification of differentially-expressed genes in human gastric carcinomas. Proteomics 5: 3205-3213, 2005. 
10. Bai Z, Ye Y, Chen D, et al: Homeoprotein CDX2 and nuclear PTEN expression profiles are related to gastric cancer prognosis. APMIS 115: 1383-1390, 2007.

11. Chan C, Ko C, Chang J, et al: Subcellular and functional proteomic analysis of the cellular responses induced by Helicobacter pylori. Mol Cell Proteomics 5: 702-713, 2006.

12. Cho H, Baek K, Park S, et al: RhoGDI2 expression is associated with tumor growth and malignant progression of gastric cancer. Clin Cancer Res 15: 2612-2619, 2009.

13. Zhang J, Jiang Y, Jia Z, et al: Association of elevated GRP78 expression with increased lymph node metastasis and poor prognosis in patients with gastric cancer. Clin Exp Metastasis 23: 401-410, 2006

14. Wang X, Liao J, Liu G, Wang X and Shang H: Co-expression of heat shock protein 70 and glucose-regulated protein 94 in human gastric carcinoma cell line BGC-823. World J Gastroenterol 11: 3601-3604, 2005.

15. Lee H, Lee H, Kim H, Yang H and Kim W: Tumour suppressor gene expression correlates with gastric cancer prognosis. J Pathol 200: 39-46, 2003.

16. Marrelli D, Pinto E, De Stefano A, Farnetani M, Garosi L and Roviello F: Clinical utility of CEA, CA 19-9, and CA 72-4 in the follow-up of patients with resectable gastric cancer. Am J Surg 181: 16-19, 2001.

17. Zhang J, Kang B, Tan X, et al: Comparative analysis of the protein profiles from primary gastric tumors and their adjacent regions: MAWBP could be a new protein candidate involved in gastric cancer. J Proteome Res 6: 4423-4432, 2007.

18. Ryu J, Kim H, Lee Y, et al: The proteomics approach to find biomarkers in gastric cancer. J Korean Med Sci 18: 505-509, 2003.

19. He Q, Cheung Y, Leung S, Yuen S, Chu K and Chiu J: Diverse proteomic alterations in gastric adenocarcinoma. Proteomics 4: 3276-3287, 2004.

20. Ebert M, Krüger S, Fogeron M, et al: Overexpression of cathepsin B in gastric cancer identified by proteome analysis. Proteomics 5: 1693-1704, 2005.

21. Wang Z, Hao Y and Lowe A: The adenocarcinoma-associated antigen, AGR2, promotes tumor growth, cell migration, and cellular transformation. Cancer Res 68: 492-497, 2008.

22. Fritzsche F, Dahl E, Pahl S, et al: Prognostic relevance of AGR2 expression in breast cancer. Clin Cancer Res 12: 1728-1734, 2006.

23. Zweitzig D, Smirnov D, Connelly M, Terstappen L, O'Hara S and Moran E: Physiological stress induces the metastasis marker AGR2 in breast cancer cells. Mol Cell Biochem 306: 255-260, 2007.

24. Zhang Y, Forootan S, Liu D, et al: Increased expression of anterior gradient-2 is significantly associated with poor survival of prostate cancer patients. Prostate Cancer Prostatic Dis 10: 293-300, 2007.
25. Feo S, Arcuri D, Piddini E, Passantino R and Giallongo A: ENO1 gene product binds to the c-myc promoter and acts as a transcriptional repressor: Relationship with Myc promoterbinding protein 1 (MBP-1). FEBS Lett 473: 47-52, 2000.

26. Niklinski J and Furman M: Clinical tumour markers in lung cancer. Eur J Cancer Prev 4: 129-138, 1995.

27. Takashima M, Kuramitsu Y, Yokoyama Y, et al: Overexpression of alpha enolase in hepatitis $C$ virus-related hepatocellular carcinoma: Association with tumor progression as determined by proteomic analysis. Proteomics 5: 1686-1692, 2005.

28. DerMardirossian C and Bokoch G: GDIs: Central regulatory molecules in Rho GTPase activation. Trends Cell Biol 15: 356-363, 2005.

29. Kwon K, Park E, Ryu D and Park B: D4-GDI is cleaved by caspase-3 during daunorubicin-induced apoptosis in HL-60 cells. Exp Mol Med 34: 32-37, 2002.

30. Ni M and Lee A: ER chaperones in mammalian development and human diseases. FEBS Lett 581: 3641-3651, 2007.

31. Chen X, Ding Y, Liu C, Mikhail S and Yang C: Overexpression of glucose-regulated protein 94 (GRP94) in esophageal adenocarcinomas of a rat surgical model and humans. Carcinogenesis 23: 123-130, 2002.

32. Iizuka N, Tsunedomi R, Tamesa T, et al: Involvement of c-mycregulated genes in hepatocellular carcinoma related to genotypeC hepatitis B virus. J Cancer Res Clin Oncol 132: 473-481, 2006.

33. Egler R, Fernandes E, Rothermund K, et al: Regulation of reactive oxygen species, DNA damage, and c-myc function by peroxiredoxin 1. Oncogene 24: 8038-8050, 2005.

34. Shi Y, Chen J, Weng C, et al: Identification of the proteinprotein contact site and interaction mode of human VDAC1 with Bcl-2 family proteins. Biochem Biophys Res Commun 305: 989-996, 2003.

35. Abu-Hamad S, Sivan S and Shoshan-Barmatz V: The expression level of the voltage-dependent anion channel controls life and death of the cell. Proc Natl Acad Sci USA 103: 5787-5792, 2006.

36. Shen W, Balajee A, Wang J, et al: Essential role for nuclear pten in maintaining chromosomal integrity. Cell 128: 157-170, 2007.

37. Li N, Zhang J, Liang Y, et al: A controversial tumor marker: Is SM22 a proper biomarker for gastric cancer cells? J Proteome Res 6: 3304-3312, 2007.

38. Wilkins M, Appel R, Van Eyk J, et al: Guidelines for the next 10 years of proteomics. Proteomics 6: 4-8, 2006. 